\title{
Solos do brejo paraibano: Influências do clima, do relevo e do material de origem
}

\author{
Brejo paraibano soils: Climate, relief and source material influences \\ Suelos del paraibano brejo: Influencias del clima, relieve y material de origen
}

\section{Resumo}

O Brejo Paraibano está localizado em cima do planalto da Borborema, onde ocorre o clima quente e úmido, influenciado principalmente pela formação de chuvas orográficas. Nesse cenário de condições específicas, ainda há poucas informações a respeito das influências do clima, do relevo e do material de origem na formação do solo. Diante do exposto, este trabalho objetivou caracterizar e classificar as manchas de solos mais representativas em áreas de floresta nativa dos municípios de Areia-PB (P1) e Alagoa Nova-PB (P2), na microrregião do Brejo Paraibano. Classificados como Argissolo (P1) e Latossolo (P2), os solos dos perfis estudados apresentaram predomínio da fração areia (> $680 \mathrm{~g}$ $\mathrm{kg}^{-1}$ ), com textura variando de argiloarenosa a muito argilosa (P1) e argilosa à argilosarenosa (P2), respectivamente. Os valores de $\mathrm{pH}$ em água variaram de 4,7 a 5,6 para ambos os solos, enquanto os valores de capacidade de troca catiônica variaram de 14,4 a $31,7 \mathrm{cmol}_{\mathrm{c}} \mathrm{dm}^{-3}$ no $\mathrm{P} 1$ e de 11,8 a $13,1 \mathrm{cmol}_{\mathrm{c}} \mathrm{dm}^{-3}$ no $\mathrm{P} 2$, com maiores valores dos íons $\mathrm{H}^{+}$e $\mathrm{Ca}^{2+}$ no P1. Os horizontes subsuperficiais do P1 e P2 são distróficos, evidenciando caráter úmbrico para o P1 e para o P2 caráter típico. O P1 apresentou argila de alta atividade em relação ao P2. Em todos os horizontes de P1 e P2 verificaramse atributos morfológicos, físicos, químicos e mineralógicos condicionados pelo material de origem e relevo, demonstrando a influência desses fatores na pedogênese.

Palavras-chave: SiBCS; Taxonomia de solos; Horizonte diagnóstico de superfície.

\begin{abstract}
The soils of the micro-region of Brejo Paraibano are the result of weathering processes and pedogenesis, under the action of formation factors (source material, living organisms, climate, topography and time), taking place preferably in hot and humid regions, with strong wavy relief and predominance of Argisol, Latosol, Alfisol and Entisol classes. The objective of this study was to characterize and classify the soils of Legal Reserve Areas developed and representative of the cities of Areia-PB (P1) and Alagoa Nova-PB (P2), located in the microregion of Brejo Paraibano. Classified as Argisol (P1) and Latosol (P2), soils of the studied profiles showed predominance of sand fraction (> 680 $\mathrm{g} \mathrm{kg}^{-1}$ ), texture ranging from sand clay to clay (P1) and clay to sand clay (P2), respectively. The $\mathrm{pH}$ in water ranging from 4.7 to 5.6 in both soils, while the cation exchange capacity values ranging from 14.4 to $31.7 \mathrm{cmol}_{\mathrm{c}} \mathrm{dm}^{-3}$ in the $\mathrm{P} 1$, and from 11.8 to $13.1 \mathrm{cmol}_{\mathrm{c}} \mathrm{dm}^{-3}$ in $\mathrm{P} 2$, with higher values of $\mathrm{H}^{+}$and $\mathrm{Ca}^{2+}$ ions on $\mathrm{P} 1$. The subsurface horizons of $\mathrm{P} 1$
\end{abstract}


and P2 are dystrophic, showing umbric carater for P1, while the P2 showed typical carater. The P1 presented high activity clay relative to P2. In all horizons P1 and P2 there were morphological, physical, chemical and mineralogical attributes conditioned by the parental material and relief, demonstrating the influence of these factors in pedogenesis.

Keywords: Mineralogy; Soil taxonomy; Horizon surface diagnosis.

\section{Resumen}

Brejo Paraibano se ubica en lo alto de la meseta de Borborema, donde se da el clima cálido y húmedo, influenciado principalmente por la formación de lluvias orográficas. En este escenario de condiciones específicas, aún existe poca información sobre las influencias del clima, el relieve y el material fuente en la formación del suelo. Dado lo anterior, este estudio tuvo como objetivo caracterizar y clasificar los parches de suelo más representativos en áreas de bosque nativo de los municipios de Areia-PB (P1) y Alagoa Nova-PB (P2), en la microrregión Brejo Paraibano. Clasificados como Argisol (P1) y Latosol (P2), los suelos de los perfiles estudiados mostraron un predominio de la fracción arena (> $680 \mathrm{~g} \mathrm{~kg}^{-1}$ ), con una textura que varió de arcilloso a muy arcilloso (P1) y arcilloso a arcilloso arenoso (P2), respectivamente. Los valores de $\mathrm{pH}$ en agua variaron de 4.7 a 5.6 para ambos suelos, mientras que los valores de capacidad de intercambio catiónico variaron de 14.4 a $31.7 \mathrm{cmol}_{\mathrm{c}} \mathrm{dm}^{-3}$ en P1 y de 11.8 a $13,1 \mathrm{cmol}_{\mathrm{c}} \mathrm{dm}^{-3}$ en P2, con valores más altos de iones $\mathrm{H}^{+}$y $\mathrm{Ca}^{2+}$ en $\mathrm{P} 1$. Los horizontes subsuperficiales de $\mathrm{P} 1$ y $\mathrm{P} 2$ son distróficos, mostrando carácter úmbrico para P1 y carácter típico para P2. P1 presentó arcilla con alta actividad en relación a P2. En todos los horizontes de P1 y P2 se encontraron atributos morfológicos, físicos, químicos y mineralógicos condicionados por el material fuente y relieve, demostrando la influencia de estos factores en la pedogénesis.

Palabras clave: SiBCS; Taxonomía de suelos; Horizonte diagnóstico de superficie.

\section{Introdução}

A microrregião do Brejo Paraibano apresenta clima do tipo quente e úmido (As’), com chuvas no período de outono e inverno, precipitação anual de 1.400 a 1.600 mm (Brasil, 1972) e relevo fortemente ondulado com áreas de vegetação primária de Floresta Ombrófila Aberta. As características e/ou propriedade e classificação do solo em Área de Reserva Legal fornece informações do solo em condições naturais (sem influência antrópica), a respeito da ciclagem de $\mathrm{C}$ e de nutrientes, retenção e infiltração de água, difusão de gases, penetração das raízes e capacidade do solo na resistência à erosão, fatores estes que são determinantes para o crescimento das plantas (Portugal et al., 2010). Estas informações também podem ser utilizadas como referência em trabalhos realizados em perfis localizados em áreas antropizadas.

Independente da cobertura vegetal em determinada área, se faz necessário conhecer e compreender a composição e a dinâmica dos processos internos do solo, pois afinal, a classificação e a relação solo - planta dependem das propriedades químicas, físicas e mineralógicas do solo (Santos et al., 2010a). Muito mais do que suporte físico para as plantas, o solo é o meio onde ocorrem reações e processos determinantes do sucesso ou insucesso da recuperação ou estabelecimento de um novo bioma. Segundo Yao et al. (2013) compreender e avaliar a qualidade do solo tem cada vez mais importância, em virtude da sustentabilidade desse recurso natural.

O desenvolvimento pedogenético dos solos reflete a atuação dos diferentes fatores de formação. Atuando em conjunto, com graus de dominância distintos, o clima, o material de origem, o relevo, o tempo, determinam as diferentes feições pedológicas dos solos. As principais informações dos ambientes evidenciam características bem peculiares, que podem estar associadas a vários processos, como presença de argila iluvial e compostos orgânicos poucos polimerizados, presença de óxido de Fe e argila dispersa e o adensamento por dessecação resultante da alteração da estrutura do solo pela alternância de ciclos de umedecimento e secagem (Rezende, 2000; Lima Neto et al., 2009).

O estudo morfológico é essencial para se caracterizar a fertilidade, que ocorre em decorrência da ciclagem e disponibilidade de cada nutriente no solo, e baseado em princípios conservacionistas, pode-se manter a proteção do solo em decorrência da cobertura vegetal que ocasiona um equilíbrio entre temperatura e umidade do solo, favorecendo altos teores de matéria orgânica, melhorando a sua qualidade, beneficiando o armazenamento de C nesse compartimento (Clemente et al., 2012), aumentando a CTC (Jiménez Becker et al., 2010) e disponibilizando nutrientes, elevando a atividade e a biomassa microbiana (Bravo et al., 2012), complexando elementos tóxicos e micronutrientes (Clemente et al., 2012), favorecendo uma menor 
densidade, maior infiltração e retenção de água, e melhorando a agregação das partículas do solo.

Mesmo com a existência de informações acerca dos solos da região do Brejo Paraibano, foram fundamentais a classificação dos solos de Reserva Legal para fins de comparação das características em função do uso e manejo de solos de localidades próximas, assim como, o levantamento exploratório para a realização da caracterização dos diferentes solos representativos em uma amplitude detalhada, já que não existem informações concretas a respeito de toda morfologia dos solos destas propriedades.

Este trabalho teve por objetivo caracterizar e classificar dois solos localizados em área de Reserva Legal, representativos da microrregião do Brejo Paraibano, e para isso, foram estudados dois perfis, P1 e P2, localizados respectivamente em Areia-PB e Alagoa Nova-PB, os quais foram morfologicamente descritos, e cujos horizontes foram caracterizados quanto às propriedades químicas, físicas e mineralógicas.

\section{Metodologia}

\subsection{Localização e características gerais da área em estudo}

$\mathrm{O}$ trabalho foi realizado em áreas de floresta nativa localizadas nos municípios de Areia-PB, perfil 1 (P1), e Alagoa Nova-PB, perfil 2 (P2), estado da Paraíba, Brasil (Figura 1). A região tem clima de Zona Tropical com verão seco, precipitação anual entre 1.300 e $1.600 \mathrm{~mm}$, temperaturas médias anuais entre 22 e $24{ }^{\circ} \mathrm{C}$ (Alvares et al., 2013) e relevo forte ondulado (declividade de 20 a 45\%).

Figura 1. Mapa de localização dos perfis P1 e P2 de solos representativos da microrregião do Brejo Paraibano, localizados nos municípios de Areia-PB (esquerda) e Alagoa Nova-PB (direita).

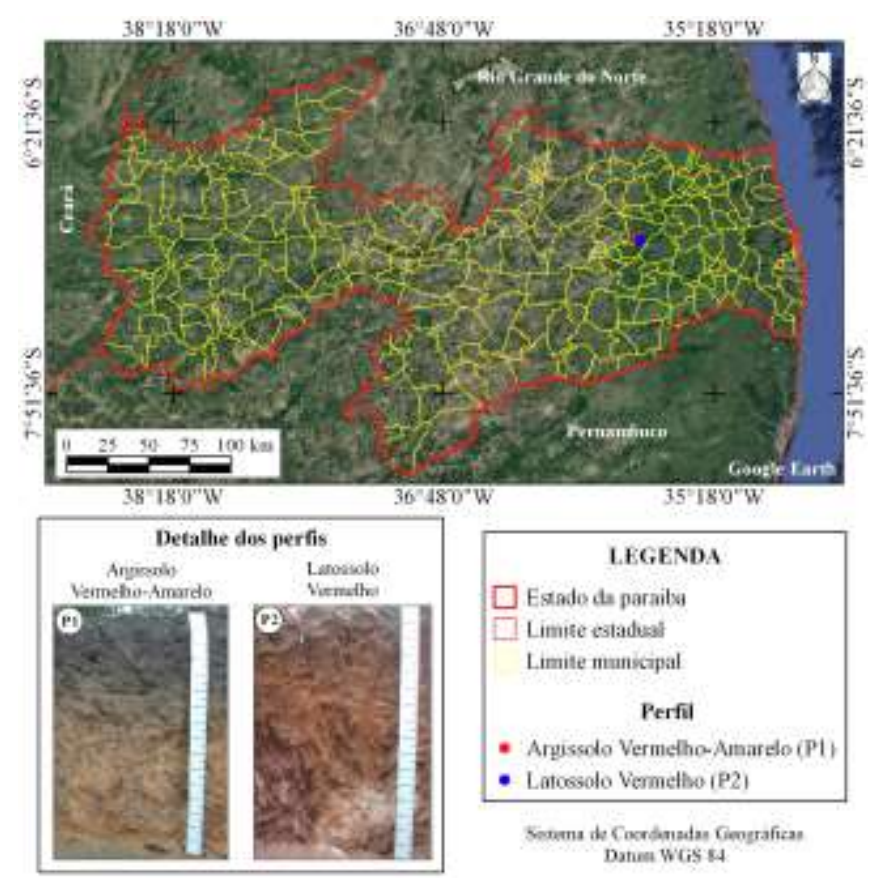

Fonte: autores.

\subsection{Identificação e classificação dos solos}

O processo de identificação e classificação dos solos foi realizado em 3 etapas: 
$1^{\text {a) }}$ Abertura das trincheiras e coleta de amostras de solo nos horizontes de cada perfil (Santos et al., 2013).

$2^{\mathrm{a}}$ ) Realização das análises químicas, físicas e mineralógicas do solo. As análises químicas (pH em água, $\mathrm{P}, \mathrm{K}^{+}, \mathrm{Ca}^{2+}, \mathrm{Mg}^{2+}, \mathrm{Na}^{+}$, $\mathrm{Al}^{3+}, \mathrm{H}+\mathrm{Al}$ e C orgânico total) e físicas (granulometria e densidade das partículas), foram realizadas com as metodologias da EMBRAPA (1997), na Universidade Federal da Paraíba (Areia-PB), laboratórios de Matéria Orgânica e Física do Solo. As análises mineralógicas foram realizadas por difratometria de raios-X (DRX) nos horizontes e suas frações granulométricas (areia fina, silte e argila), com os difratômetros de raios-X Bruker D2 Phaser, do Instituto Nacional do Semiárido, Laboratório de raios$\mathrm{X}$.

$3^{\text {a) }}$ Classificação dos perfis dos solos com base no Sistema Brasileiro de Classificação de Solos (EMBRAPA, 2013), por apresentar um enfoque agrícola.

Os atributos morfológicos e físicos não foram analisados no horizonte $\mathrm{O}$, visto que este horizonte é considerado diagnóstico apenas na ordem denominada de Organossolos (Santos et al., 2018), que não ocorre na região em estudo.

\section{Resultados e Discussão}

A seguir, são apresentadas as variações morfológicas, os atributos físicos e químicos dos dois perfis de solos. Como estes são solos moderadamente drenados que pertencem a classes distintas, os mesmos são resultantes de fatores e processos pedogenéticos diferenciados que lhes dão características variadas, P1 (Argissolo) com material de origem proveniente de saprolito de rochas gnaisses, granito e o P2 (Latossolo) de Pseudo autóctone.

\subsection{Aspectos morfológicos}

Em ambos os perfis P1 e P2, nos horizontes superficiais e subsuperficiais constatou-se grande predominância de estruturas do tipo granular, blocos angulares e subangulares (Tabela 1).

Verificou-se o predomínio do matiz 7,5YR com altos valores do croma (valores entre 2 e 8) para o P1, denotando cor vermelha-amarela, diferentemente do P2 que apresentou-se com matizes mais avermelhadas (2,5YR) e altos valores do croma (6 a 8), indicando a cor vermelha. As características do P2 são decorrentes dos teores mais altos e da natureza dos óxidos de Fe presentes no material originário. Salienta-se que estas cores são comuns nos solos do tipo Argissolo e Latossolo (EMBRAPA, 2013).

Em P1 verificaram-se mosqueados vermelhos em quantidade abundante a partir dos horizontes BA e B, com tamanho médio e contraste distinto, e coloração vermelha amarela que indica a presença de oxihidróxido de Fe (hematita e goethita), enquanto em P2 não houve presença de mosqueados entre os horizontes, indicando que não há oscilação do nível do lençol freático. Ribeiro et al. (2012) descrevem a presença de mosqueados no solo, como indicativo, que revela geralmente condições de drenagem restrita sendo observados em solos que apresentam horizonte de baixa permeabilidade e, ou, naqueles que estão localizados em posições de paisagem que favorecem a oscilação do nível do lençol freático. Essas cores são indicativas da ocorrência do material mineral, o qual é fornecedor de Fe e Mg (EMBRAPA, 2013). 
Tabela 1. Principais atributos morfológicos de P1 e P2, localizados nos municípios de Areia-PB e Alagoa-Nova-PB, respectivamente.

\begin{tabular}{|c|c|c|c|c|c|c|c|c|}
\hline \multirow{2}{*}{ Horiz. } & \multirow{2}{*}{$\begin{array}{c}\text { Prof. } \\
\mathbf{c m} \\
\end{array}$} & \multicolumn{2}{|c|}{ Cor $^{1}$} & \multirow{2}{*}{ Estrutura } & \multicolumn{3}{|c|}{ Consistência } & \multirow{2}{*}{ Textura } \\
\hline & & Úmida & Seca & & Seca & Úmida & Molhada & \\
\hline \multicolumn{9}{|c|}{ Perfil 1 (P1) } \\
\hline A & $10-20$ & $\begin{array}{c}7,5 \text { YR- } \\
3 / 2\end{array}$ & $\begin{array}{c}7,5 \\
\text { YR-4,2 }\end{array}$ & $\begin{array}{l}\text { mod., peq. a } \\
\text { méd., a gr., bl. } \\
\text { ang. e sub. }\end{array}$ & LD & $\mathrm{F}$ & $\mathrm{Pl}$ e Pe & Argilo arenosa \\
\hline $\mathrm{AB}$ & $20-40$ & $\begin{array}{c}7,5 \text { YR- } \\
3 / 2\end{array}$ & $\begin{array}{c}7,5 \\
\text { YR-5,2 }\end{array}$ & $\begin{array}{c}\text { mod., peq. a } \\
\text { méd, a gr., bl. } \\
\text { ang. e sub. }\end{array}$ & LD & $\mathrm{F}$ & $\mathrm{Pl}$ e Pe & Argilosa \\
\hline BA & $40-60$ & $\begin{array}{c}7,5 \text { YR- } \\
3 / 2\end{array}$ & $\begin{array}{c}7,5 \\
\text { YR-4,2 }\end{array}$ & $\begin{array}{l}\text { mod., peq. a } \\
\text { méd., a gr., bl. } \\
\text { ang. e sub. }\end{array}$ & LD & $\mathrm{F}$ & $\mathrm{Pl}$ e Pe & Argilo arenosa \\
\hline B & $60-160$ & $\begin{array}{c}7,5 \text { YR- } \\
4 / 4\end{array}$ & $\begin{array}{c}7,5 \\
\text { YR-6/8 }\end{array}$ & $\begin{array}{l}\text { mod., peq. a } \\
\text { méd., a gr., bl. } \\
\text { ang. e sub. }\end{array}$ & LD & $\mathrm{F}$ & $\mathrm{Pl}$ e $\mathrm{Pe}$ & Muito Argilosa \\
\hline \multicolumn{9}{|c|}{ Perfil 2 (P2) } \\
\hline A & $0-20$ & $\begin{array}{c}2,5 \text { YR- } \\
3 / 6\end{array}$ & $\begin{array}{c}2,5 \\
\text { YR-4/6 }\end{array}$ & $\begin{array}{l}\text { mod., peq. a } \\
\text { méd., a gr., bl. } \\
\text { ang. e sub. }\end{array}$ & LD & $\mathrm{Fe} \mathrm{Fi}$ & MPl e MPe & Argilosa \\
\hline BA & $20-60$ & $\begin{array}{c}2,5 \text { YR- } \\
4 / 8\end{array}$ & $\begin{array}{c}2,5 \\
\text { YR-5/8 }\end{array}$ & $\begin{array}{c}\text { mod., peq. a } \\
\text { méd., a gr., bl. } \\
\text { ang. e sub. }\end{array}$ & MD & $\mathrm{F}$ e Fi & MPl e MPe & Argilosa \\
\hline B & $60-160$ & $\begin{array}{c}2,5 \text { YR- } \\
3 / 6\end{array}$ & $\begin{array}{c}2,5 \\
\text { YR-3/6 }\end{array}$ & $\begin{array}{l}\text { mod., peq. a } \\
\text { méd., a gr., bl. } \\
\text { ang. e sub. }\end{array}$ & LD & $\mathrm{F}$ e $\mathrm{Fi}$ & MPl e MPe & Franco-Argilosa \\
\hline
\end{tabular}

Horiz.: Horizonte; Prof.: Profundidade; ${ }^{1}$ escala de Munsell; mod.: moderada ; peq.: pequena; med.: média; gr.: grande; bl.: blocos; ang.: angulares; sub.: subangulares; LD: ligeiramente dura; MD: Muito dura; F: Friável; Fi: Firme; Pl: plástica; MPl: muito plástica; Pe: pegajosa; MPe: muito pegajosa. Fonte: Autores.

A consistência, quando seca, foi ligeiramente dura no P1 e P2 em diferentes profundidades. Quando úmida, a consistência analisada nos horizontes esteve friável no P1, e variou de friável a firme no P2. Quando molhada, a consistência era plástica e pegajosa no P1 e muito plástica e muito pegajosa no P2. Tratando-se de solos muito intemperizados, desenvolvidos, profundos e drenados, em termos de consistência, relatam que é indispensável esse conhecimento devido às implicações de ordem prática (MOTA et al., 2008).

O perfil 1 (P1) apresentou textura de argilo arenosa a muito argilosa, enquanto o P2, argilosa a franco argilosa (Tabela 1). Em face ao exposto, constata-se que no horizonte A do P2 tende a ter maior capacidade de retenção de água em relação ao mesmo horizonte do P1.

\subsection{Atributos físicos}

Em todos os perfis os teores de argila foram superiores a $280 \mathrm{~g} \mathrm{~kg}^{-1}$, em razão da natureza do material de origem de rochas de gnaisse e granito transportados à pequena distância (Tabela 2). No P1 observaram-se os maiores teores de argila nos horizontes subsuperficiais B e BA, onde se identificou, segundo os critérios estabelecidos pelo SiBCS (EMBRAPA, 2013), um horizonte B textural, diferentemente do P2, caracterizado simplesmente por B. O grau de floculação (GF) nos perfis foi alto, variando de 83 até $100 \%$, tanto nos horizontes superficiais, como subsuperficiais, indicando forte presença de argila. Com relação ao GF, a proporção de argila indica que está se encontra floculada (EMBRAPA, 2011). Contudo, o horizonte B do P1, teve menor GF e maior teor de argila que o horizonte B do P2, o que pode estar associado ao processo de iluviação, responsável pelo acúmulo de argila no horizonte $\mathrm{B}$. 
Os valores de densidade do solo (Ds) variaram de 1,35 a 1,48 $\mathrm{g} \mathrm{cm}^{-3}$ no P1 e de 1,36 a 1,52 $\mathrm{g} \mathrm{cm}^{-3}$ no P2, sendo verificada correspondência entre os valores mais elevados de Ds e os valores de areia ou argila, observados no P1 (Tabela 3). Os solos ou camadas mais arenosas apresentam valores elevados de densidade observados nos Argissolos e Latossolos, onde os horizontes. A desses solos possuem textura menos argilosa que o horizonte B, proporcionando maior densidade, o que corrobora com os resultados de Ferreira (2010) que estudou as propriedades físicas e químicas de um solo arenoso da região sudoeste do Rio Grande do Sul. Observam-se em solos arenosos, valores maiores de densidades do solo, quando comparados aos argilosos, devido, possivelmente, ao alto peso das partículas de quartzo e menor conteúdo de matéria orgânica (AGUIAR, 2008). Os valores de Ds do P1, que variaram de 1,35 a 1,48 $\mathrm{g} \mathrm{cm}^{-3}$ e de 1,36 a $1,52 \mathrm{~g} \mathrm{~cm}^{-3}$ no P2, refletem nestes solos algumas das características do seu sistema poroso, pois como as raízes das plantas encontram-se nesses locais, admite-se que qualquer alteração nesse sistema possa interferir no seu desenvolvimento. É importante destacar que os valores mais elevados de Ds para o P1 (1,48 g $\left.\mathrm{cm}^{-3}\right)$ e P2 $\left(1,52 \mathrm{~g} \mathrm{~cm}^{-3}\right)$ podem limitar o desenvolvimento radicular das plantas e a capacidade de armazenamento de água no solo.

Os valores de densidade de partícula (Dp) do P1 variaram de 2,54 $\mathrm{g} \mathrm{cm}^{-3}$ no horizonte A até $2,67 \mathrm{~g} \mathrm{~cm}^{-3}$ no horizonte $\mathrm{B}$, já em P2 variaram de $2,60 \mathrm{~g} \mathrm{~cm}^{-3}$ no horizonte A até $2,83 \mathrm{~g} \mathrm{~cm}^{-3}$ no horizonte B. A maior parte dos valores de Dp dos horizontes nos dois perfis de solos estudados está acima da média universal que é $2,65 \mathrm{~g} \mathrm{~cm}^{-3}$, indicando a presença de partículas densas como o quartzo, mineral este que é considerado predominante na maioria dos solos se comparado aos outros minerais existentes. A porosidade nos horizontes dos solos variou de $0,42 \mathrm{a} 0,48 \mathrm{dm}^{3} \mathrm{dm}^{-3}$, estando na maioria dentro dos limites propostos por Kiehl (1979) para solos de textura média a argilosa, cuja porosidade total varia de 0,40 a $0,60 \mathrm{dm}^{3} \mathrm{dm}^{-3}$, ou para solos mais arenosos nos quais a variação se encontra entre 0,35 e $0,50 \mathrm{dm}^{3} \mathrm{dm}^{-3}$. Já com relação aos teores da fração argila, ocorre comportamento inverso ao da fração areia, havendo tendência de aumento gradual, excetuando-se o horizonte B do P2, conforme se apresenta a seguir: $390 \mathrm{~g} \mathrm{~kg}^{-1}$ (horizonte A), $430 \mathrm{~g} \mathrm{~kg}^{-1}$ (horizonte $\mathrm{AB}$ ), $390 \mathrm{~g} \mathrm{~kg}^{-1}$ (horizonte BA) e $627 \mathrm{~g} \mathrm{~kg}^{-1}$ (horizonte B) para o P1, enquanto o P2 apresentou $372 \mathrm{~g} \mathrm{~kg}^{-1}$ (horizonte A), $481 \mathrm{~g} \mathrm{~kg}^{-1}$ (horizonte BA) e $371 \mathrm{~g} \mathrm{~kg}^{-1}$ (horizonte B). Essas variações dos teores de argilas nos horizontes dos perfis P1 e P2 estão relacionadas com a Lessivagem, ou seja, movimento em suspensão das partículas da fração argila, óxidos e compostos orgânicos no interior do solo. Como consequência, os horizontes superficiais são empobrecidos (eluviados), enquanto os horizontes subsuperficiais são enriquecidos (iluviados) em argila (KER et al., 2012).

As análises morfológicas (Tabela 1) e físicas (Tabela 2) do P1 referem-se a um Argissolo. Este solo apresentou teores de areia mais elevados nos horizontes A e BA, $498 \mathrm{e} 505 \mathrm{~g} \mathrm{~kg}^{-1}$, respectivamente, que vão diminuindo à medida que aumenta a profundidade do solo. Nota-se que nesses horizontes, A, BA e AB, há o predomínio de areias fina e muito fina proporcionando uma maior porosidade e maior retenção de água, apesar da baixa quantidade de argila presente nesses horizontes em relação aos de maior profundidade. Há pouca areia fina e areia muito fina no horizonte $\mathrm{B}$, com predominância de argila em maior profundidade no horizonte $\mathrm{B}$. 


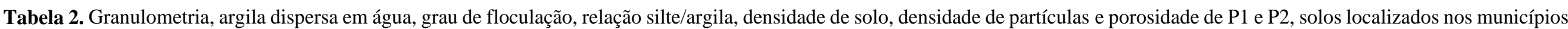
de Areia-PB e Alagoa Nova-PB, respectivamente.

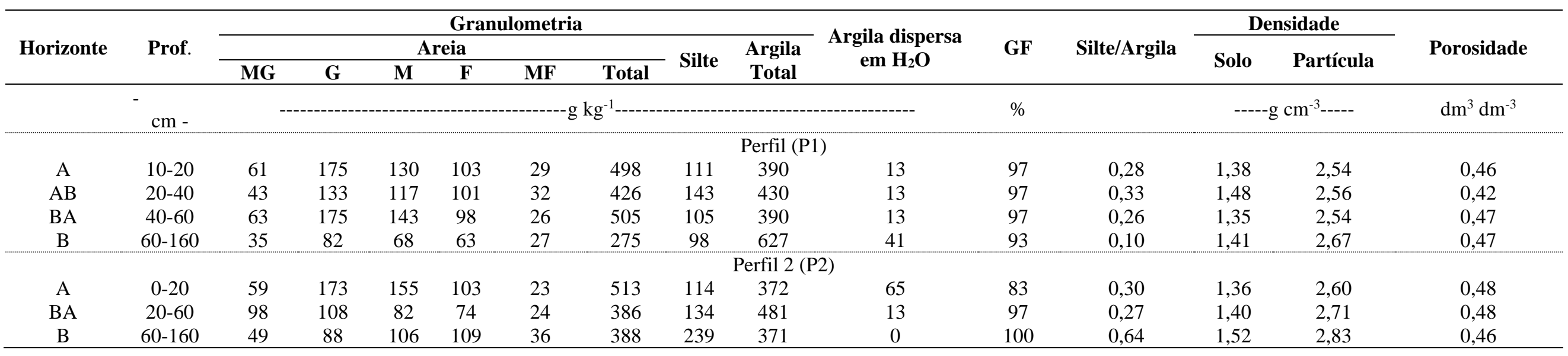

MG: areia muito grossa; G: areia grossa; M: areia média; F: areia fina; MF: areia muito fina; GF: grau de floculação. Fonte: Autores.

Tabela 3. Atributos químicos de P1 e P2, solos localizados nos municípios de Areia-PB e Alagoa Nova-PB, respectivamente.

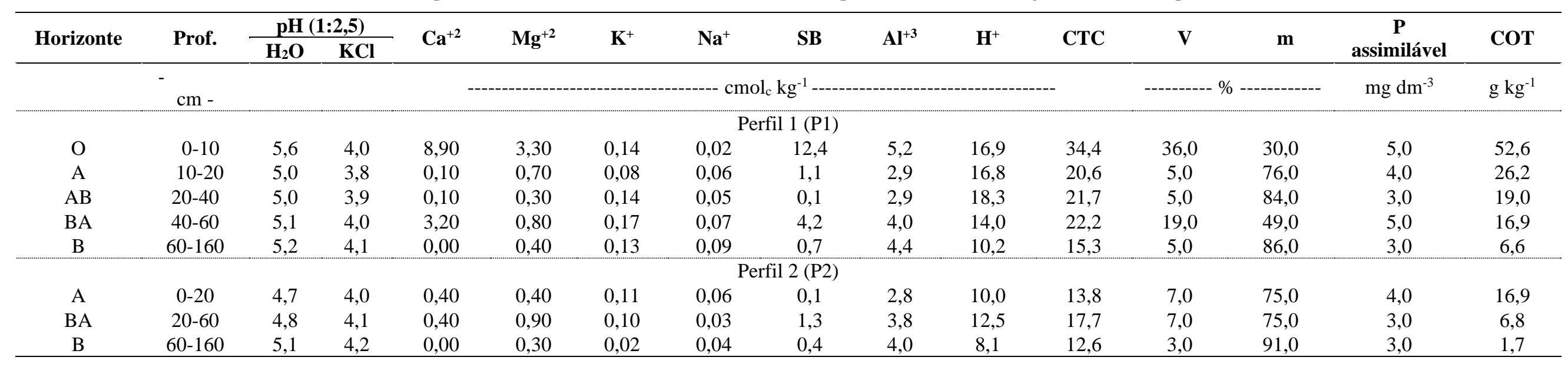

SB: soma de bases; CTC: Capacidade de Troca Catiônica a pH 7; V: saturação por bases; m: saturação por alumínio; COT: Carbono Orgânico Total. Fonte: Autores. 
De acordo com Santos et al. (2012), teores de areia fina e areia muito fina grandes contribuem para um aumento na retenção e disponibilidade de água no perfil.

As areias dos horizontes A e BA são constituídas predominantemente por frações do tipo muito grossa e grossa. Essa predominância de frações grosseiras de areia nesses horizontes são características indicadoras de que os processos intempéricos atuantes nesse solo não foram capazes de promover marcante fragmentação dessas frações. Percebe-se que não houve variação nos teores da fração silte ao longo do P1, que vai diminuindo com o aumento da profundidade, de $111 \mathrm{~g} \mathrm{~kg}^{-1}$ no horizonte $\mathrm{A}$ e $98 \mathrm{~g} \mathrm{~kg}^{-1}$ no B, com exceção do horizonte $\mathrm{AB} 143 \mathrm{~g} \mathrm{~kg}^{-1}$.

O acúmulo de argila no horizonte B é um dos atributos definidores dos Argissolos, pois, à medida que há um aumento significativo de argila em relação aos horizontes subjacentes, aumenta a possibilidade de ocorrência de processos erosivos. No entanto, a maioria dos solos desta classe apresenta um incremento no teor de argila do horizonte superficial A para o horizonte B, com ou sem decréscimo, para baixo no perfil (EMBRAPA, 2013).

Já no P2 os teores de silte são altos, crescendo de 114 a $239 \mathrm{~g} \mathrm{~kg}^{-1}$. Com relação à argila, observa-se um aumento dessa fração à medida que aumenta a profundidade do solo, de $372 \mathrm{~g} \mathrm{~kg}^{-1}$ no horizonte A para $481 \mathrm{~g} \mathrm{~kg}^{-1}$ no horizonte BA, exceto no horizonte B que mostrou um teor de argila menor de $371 \mathrm{~g} \mathrm{~kg}^{-1}$. O solo em estudo, classificado como um Latossolo, apresentou teor de argila maior que $35 \%$ nas três camadas avaliadas (Tabela 2). De acordo com o triângulo textural (FERREIRA, 2010) atribui-se, assim, a textura do solo como argilo arenosa, argilosa e franco argilosa.

A relação silte/argila, apresentou valor médio de 0,1 no P1 e de 0,64 no P2, sendo estes, valores considerados baixos, estando, assim, em conformidade com o elevado grau de desenvolvimento pedológico do solo. Ademais, quimicamente os teores dos elementos encontrados (Tabela 3) são condizentes com o alto grau de intemperismo desenvolvido nas unidades de mapeamento.

A porosidade total nos horizontes destes solos variou entre 0,47 e $0,48 \mathrm{dm}^{3} \mathrm{dm}^{-3}$, estando de acordo com os limites expostos por Kiehl (1979), onde, para os solos de textura média e argilosa, a porosidade total varia de $40 \%$ a $60 \%$; já para solos com textura mais arenosa, a variação se encontra entre 35\% a 60\%. É necessário ressaltar que a porosidade do solo depende de características como a textura e o teor de matéria orgânica do solo (MOS) que influenciam o tipo de estrutura; o manejo que provoca alterações no conteúdo da MOS e desagrega o solo (KÄMPF e CURI, 2012). A consistência e a estrutura mostram a participação de minerais de argila do tipo 2:1 confirmada pela difratometria de raios-X.

\subsection{Atributos químicos}

Os valores de pH em água variaram entre 5,0 e 5,6, para o P1, e de 4,7 a 5,1 para o P2 (Tabela 3). No P1, observaramse os maiores valores de $\mathrm{pH}$ paralelos ao aumento dos teores de $\mathrm{Al}^{+3}$. Provavelmente os valores de $\mathrm{pH}$ altos do $\mathrm{P} 1$ devem estar relacionados, primeiramente, ao seu material de origem (granito e gnaisse), constituído de rochas ácidas; diferentemente do P2, cujo material de origem presente é o Pseudo autóctone. Além disso, tratam-se de áreas dotadas de maior pluviosidade e

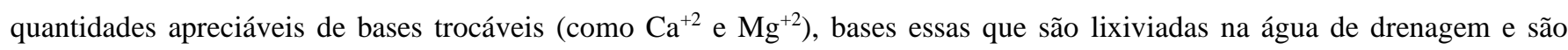
substituídas por elementos acidificantes, como H, Mn e Al (MALAVOLTA, 2006). No perfil P2, os valores de pH em água foram próximos a 5,1, com pouca variação em profundidade, sendo observada a presença de $\mathrm{Al}^{+3}$.

Os teores de $\mathrm{Ca}^{+2}$ maiores ocorreram nos horizontes superficiais, com variações de $8,90 \mathrm{cmol}_{\mathrm{c}} \mathrm{kg}^{-1}$ no horizonte A até $0,00 \mathrm{cmol}_{\mathrm{c}} \mathrm{kg}^{-1}$ no horizonte B, do P1. Enquanto no P2 variaram entre $0,40 \mathrm{cmol}_{\mathrm{c}} \mathrm{kg}^{-1}$ no horizonte $\mathrm{A} \mathrm{e} 0,00 \mathrm{cmol}_{\mathrm{c}} \mathrm{kg}^{-1}$ horizonte $\mathrm{B}$; diminuindo assim em profundidade para os dois perfis (Tabela 3). Os valores de $\mathrm{Mg}^{+2}$ no P1 $\left(3,30\right.$ a $\left.0,40 \mathrm{cmol}_{\mathrm{c}} \mathrm{kg}^{-1}\right)$ e P2 $\left(0,40\right.$ a $\left.0,30 \mathrm{cmol}_{\mathrm{c}} \mathrm{kg}^{-1}\right)$ apresentaram pouca variação em profundidade para o P1. Ainda, nos perfis $\mathrm{P} 1$ e P2 os valores de $\mathrm{Mg}{ }^{+2}$ não resultaram diferenças significativas em relação aos teores de $\mathrm{Ca}^{+2}$. Teores de $\mathrm{P}$ médios foram registrados entre os dois perfis com valores sempre acima de 3,0 $\mathrm{mg} \mathrm{dm}^{-3}$ em virtude da riqueza do material de origem, pois o P1 apresentou valores de $\mathrm{P}$ entre 
5,0 a 3,0 $\mathrm{mg} \mathrm{dm}^{-3}$ e o P2 4,0 a 3,0 $\mathrm{mg} \mathrm{dm}^{-3}$, que diferem-se entre si. Segundo Brasil (1972) os solos brasileiros, inclusive os solos do semiárido, em geral, são pobres em P devido a sua baixa concentração no material de origem. Os Latossolos do Cerrado são naturalmente pobres em P e, segundo Carneiro et al. (2009), a baixa fertilidade deve-se à natureza distrófica. Jakelaitis et al. (2008) também perceberam a natureza distrófica do Argissolo Vermelho-Amarelo, resultando em pobreza nutricional.

Guareschi e Perin (2013), em estudo das frações da matéria orgânica e das propriedades físicas e químicas em áreas de um Latossolo sob diferentes sistemas de manejo no Cerrado do Estado de Goiás, apresentaram valores de $\mathrm{Ca}^{+2} \mathrm{e} \mathrm{K}^{+}$semelhantes aos dados químicos do P1, com diferença nos teores de $\mathrm{Mg}^{+2}, \mathrm{Al}^{+3}\left(1,9 \mathrm{cmol}_{\mathrm{c} \mathrm{kg}} \mathrm{ke} \mathrm{Mg}^{+2}, 0,5 \mathrm{cmol}_{\mathrm{c} \mathrm{kg}} \mathrm{de}^{-1} \mathrm{Al}^{+3}\right.$ ), valores estes

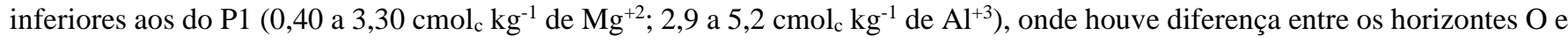
$\mathrm{B}$ em relação aos teores de $\mathrm{Mg}^{+2}$ e $\mathrm{Al}^{+3}$. O (P1) apresenta acidez elevada no horizonte $\mathrm{O}, \mathrm{A}, \mathrm{AB}$ e BA enquanto no (P2) Latossolo, a acidez é considerada média nos seus respectivos horizontes. Isso indica predomínio de cátions ácidos $(\mathrm{H}+\mathrm{Al})$ nos sítios de troca e alta saturação por $\mathrm{Al}^{+3}(\mathrm{~m}>80 \%)$ nos horizontes $\mathrm{B}$ em cada perfil. No entanto, a acidez em profundidade conjugada com elevados teores de Al trocável pode ser decorrente do processo de ferrólise. Segundo Brinkman (1970), a ferrólise é comum em solos ácidos sujeitos à redução sazonal e ricos em Fe.

Os valores de CTC do P1 mostram baixa saturação por bases (V) e baixa CTC, com exceção do horizonte O (34,7 cmolc $\mathrm{kg}^{-1}$ ), principalmente devido ao teor de CO elevado de 52,6 $\mathrm{g} \mathrm{kg}^{-1}$, o que lhe confere certa toxidez por $\mathrm{Al}^{+3}$ (Tabela 3). Quando o CO total do solo aumenta, eleva também a CTC. Como consequência há uma menor quantidade de $\mathrm{K}^{+}$na solução do solo e menor é a sua perda por lixiviação (YAMADA; ABDALLA, 2004). Quanto aos valores de V\% no P1 e P2, os horizontes diagnósticos subsuperficiais foram identificados como distróficos ( $\mathrm{V}<50 \%$ ) (EMBRAPA, 2013). No P1 os teores de CO em profundidade $\left(52,6,26,2,19,0,16,9\right.$ e $\left.6,6 \mathrm{~g} \mathrm{~kg}^{-1}\right)$ foram mais elevados comparados ao $\mathrm{P} 2\left(16,9,6,8\right.$ e 1,7 $\left.\mathrm{g} \mathrm{kg}^{-1}\right)$, o que pode estar relacionado à textura com maior teor de argila e silte, cujos valores foram $390 \mathrm{e} 111 \mathrm{~g} \mathrm{~kg}^{-1}$, respectivamente, para TFSA no P1 (Tabela 2).

Os teores baixos de P nos horizontes superficiais do P1 e P2 devem estar relacionados ao solo ser de reserva legal e no qual está extinta a prática de cultivo com o uso de resíduos de adubações fosfatadas a pelo menos 30 anos.Com relação aos elementos extraídos e obtidos do P1 e P2 por FRX, pode-se observar (Tabela 4) que o Si apresentou valores dominantes em todos os solos nas amostras de cada horizonte do P1 e P2, com maiores valores no Argissolo, especialmente no horizonte BA. Isso ocorreu devido à crosta terrestre ser formada principalmente de sílica $\left(\mathrm{SiO}_{2}\right)$ e silicatos (tetraedros e $\left.\mathrm{SiO}_{4}\right)$, aproximadamente 97\% em termos de volume, segundo Madureira Filho, Atencio e McReath (2000, p. 34). Dessa maneira, a crosta terrestre continental é formada por uma estrutura contendo basicamente $\mathrm{Si}$, $\mathrm{O}$ e $\mathrm{Al}$, cujos interstícios são preenchidos por outros elementos como $\mathrm{Fe}, \mathrm{Ca}, \mathrm{Mg}, \mathrm{Na}$, K, entre outros.

O Argissolo Vermelho-Amarelo (P1) apresentou na sua mineralogia quartzo $\left(\mathrm{SiO}_{2}\right)$, muscovita $\left(\mathrm{K}_{2} \mathrm{Al}_{4}\left[\mathrm{Si}_{6} \mathrm{Al}_{2} \mathrm{O}_{20}\right](\mathrm{OH}\right.$, $\left.\mathrm{F})_{4}\right)$, microline $\left(\mathrm{KAlSi}_{3} \mathrm{O}_{8}\right)$ e/ou ortoclase $\left(\mathrm{KAlSi}_{3} \mathrm{O}_{8}\right)$ - denominados feldspato-K, oligoclase $\left(\mathrm{NaAlSi}_{3} \mathrm{O}_{8}\right)$ - denominado feldspato-Na, hematita $\left(\mathrm{Fe}_{2} \mathrm{O}_{3}\right)$, caulinita $\left(\mathrm{Al}_{2} \mathrm{O}_{3}, 2 \mathrm{SiO}_{2} \cdot 2 \mathrm{H}_{2} \mathrm{O}\right)$ e gibbsita $\left(\mathrm{Al}(\mathrm{OH})_{3}\right)$ (Tabela 1). Como esperado, notam-se teores mais elevados de Si e Fe presentes na hematita, quartzo e muscovita, além da forte predominância do Si e do Al, respectivamente. Em relação ao Latossolo Vermelho (P2), observa-se que este apresentou na sua mineralogia quartzo $\left(\mathrm{SiO}_{2}\right)$, caulinita $\left(\mathrm{Al}_{2} \mathrm{O}_{3}\right.$, $\left.2 \mathrm{SiO}_{2} .2 \mathrm{H}_{2} \mathrm{O}\right)$, muscovita $\left(\mathrm{K} 2 \mathrm{Al} 4\left[\mathrm{Si}_{6} \mathrm{Al}_{2} \mathrm{O}_{2} \mathrm{O}\right](\mathrm{OH}, \mathrm{F})_{4}\right)$, ilita $\left(\mathrm{K}, \mathrm{H}_{3} \mathrm{O}\right)(\mathrm{Al}, \mathrm{Mg}, \mathrm{Fe})_{2}(\mathrm{Si}, \mathrm{Al}) 4 \mathrm{O}_{10}\left[(\mathrm{OH})_{2},\left(\mathrm{H}_{2} \mathrm{O}\right)\right]$, microline $\left(\mathrm{KAlSi}_{3} \mathrm{O}_{8}\right)$ e/ou Ortoclase $\left(\mathrm{KAlSi}_{3} \mathrm{O}_{8}\right)$ - denominado feldspato-K, Oligoclase $\left(\mathrm{NaAlSi}_{3} \mathrm{O}_{8}\right)$ - denominado feldspato $\mathrm{K}$, goethita $(\mathrm{FeO}(\mathrm{OH}))$, hematita $\left(\mathrm{Fe}_{2} \mathrm{O}_{3}\right)$ e gibbsita $\left(\mathrm{Al}(\mathrm{OH})_{3}\right)($ Tabela 2). Em virtude de ser uma área de mata, não sofrendo manejo, os teores $\mathrm{K}, \mathrm{Ca}$ e $\mathrm{P}$, entre outros elementos, apresentaram valores baixos.

Comparando os dois perfis, nota-se que o teor de Fe no P2 é maior que no P1 (Tabela 4), evidenciando a elevada coloração vermelha. Em relação ao Argissolo Vermelho-Amarelo, observa-se uma sensível redução do teor de Fe e como consequência desta escassa presença de óxidos de Fe, onde se evidencia estágio de intemperismo menos avançado que o 
Latossolo Vermelho. Em relação à disponibilidade de $\mathrm{P}$, percebe-se baixo valor nos dois tipos de solo, o que decorre da área ser inativa, além desse nutriente ser adsorvido na superfície dos minerais secundários por serem solos intemperizados. Farias et al. (2009) avaliando a disponibilidade de P nos solos de referência do Estado de Paraíba, concluíram que a adição de P na forma de fertilizante a solos ácidos e muito intemperizados promove a adsorção de parte desse elemento na superfície dos minerais secundários da fração argila (principalmente óxidos de $\mathrm{Fe}$ e $\mathrm{Al}$ ) e outra parte se precipita com íons $\mathrm{Fe}^{+3}$ e $\mathrm{Al}^{+3}$ presentes na solução do solo, exigindo que práticas adequadas de manejo como adubação fosfatada nesses solos sejam adotadas.

Os valores de Si variaram de 34,98 a 44,94 $\mathrm{g} \mathrm{kg}^{-1}$; Al de 21,4 a 27,21 $\mathrm{g} \mathrm{kg}^{-1} ; \mathrm{Fe}$, de 24,17 a 33,05 $\mathrm{g} \mathrm{kg}^{-1}$; e Ti de 2,97 a $3,79 \mathrm{~g} \mathrm{~kg}^{-1}$ para o P1 (Tabela 4), já para o P2, os valores de Si variaram de 27,31 a 34,4 $\mathrm{g} \mathrm{kg}^{-1}$; Al de 18,33 a 21,38 g kg-1; Fe de 40,25 a 49,31 $\mathrm{g} \mathrm{kg}^{-1}$; e Ti de 3,50 a 3,73 $\mathrm{g} \mathrm{kg}^{-1}$. De maneira geral, os altos valores desses elementos estão associados aos altos conteúdos de argila observados nos solos estudados. No P1 e P2 verificaram-se incrementos nos teores de $\mathrm{SiO}_{2}, \mathrm{Al}_{2} \mathrm{O}_{3}, \mathrm{Fe}_{2} \mathrm{O}_{3}$ nos horizontes subsuperficiais, em virtude da localização da paisagem e ao relevo forte ondulado, ocasionando drenagem moderada.

Quanto aos teores de $\mathrm{TiO}_{2}$, observou-se pouca variação, podendo-se inferir, pela uniformidade dos materiais e pelo material de origem do solo, que se trata de saprolito das rochas gnaisse e granito para o Argissolo e Pseudo autóctone para o Latossolo. 


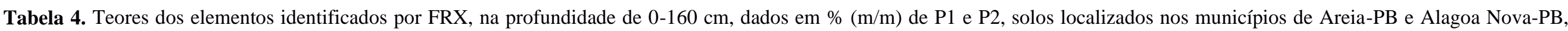
respectivamente.

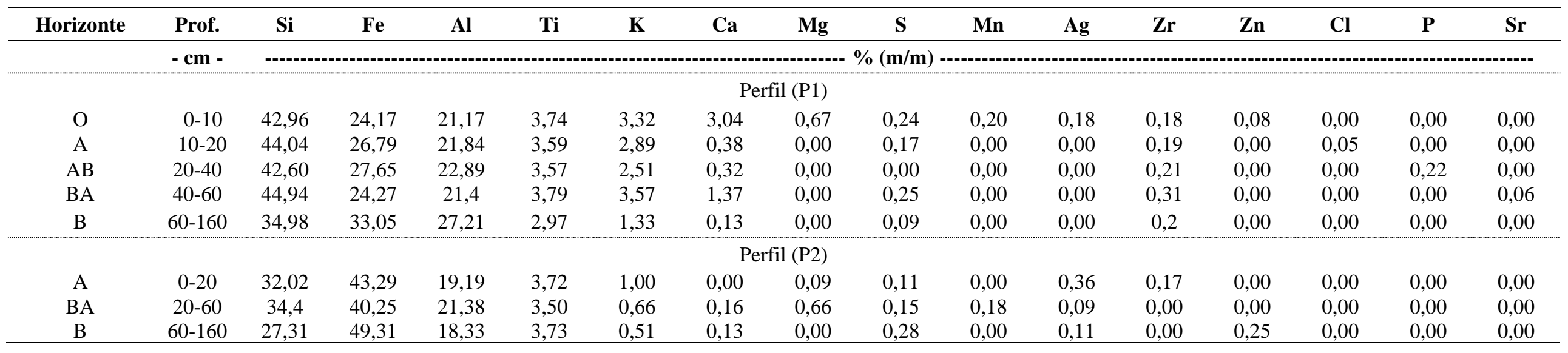

Fonte: Autores 


\section{Conclusão}

Os perfis apresentaram cores vermelho-amarelo (P1) e vermelho (P2), por estarem localizados em uma região moderadamente drenada, sendo estes seus principais atributos (morfológico e físico). Em todos os perfis observou-se o predomínio da estrutura em blocos angulares e subangulares em subsuperfície, e textura variando de argilo arenosa a muito argilosa em P1 e no P2 variando de argilosa a franco-argilosa.

Em relação aos atributos químicos e mineralógicos constataram-se solos com saturação por bases variando de 3 a $5 \%$ no horizonte B e predomínio de argilas de atividade baixa (CTC $<27 \mathrm{cmol}_{\mathrm{c}} \mathrm{kg}^{-1}$ ), com apenas o horizonte O do P1 com 34,4 $\mathrm{cmol}_{\mathrm{c}} \mathrm{kg}^{-1}$.

Com base nas análises dos atributos morfológicos, químicos, físicos e mineralógicos, constatou-se que o relevo e o material de origem exercem grande influência na gênese dos solos estudados, os quais foram classificados como Argissolo Vermelho-Amarelo distrófico úmbrico (P1) e o Latossolo Vermelho Distrófico típico (P2).

\section{Agradecimentos}

À CAPES e CNPQ pela bolsa de mestrado concedida à Universidade Federal da Paraíba (UFPB), através do Programa de Pós-Graduação em Ciência do Solo (PPGCS).

Aos Laboratórios de Matéria Orgânica, Química Instrumental e Física do Solo, da Universidade Federal da Paraíba (UFPB/CCA), Campus II, pelas análises químicas e físicas do solo.

Ao Laboratório do Departamento de Física da Universidade Federal do Ceará (UFC) pelas análises de FRX e DRX.

\section{Referências}

Alvares, C. A., Stape, J. L., Sentelhas, P. C., Gonçalves, J. L. M., Sparovek, G. (2013). Köppen's climate classification map for Brazil. Meteorologische Zeitschrift, 22, 711-728. 10.1127/0941-2948/2013/0507

Aguiar, M. I. (2008). Qualidade física do solo em sistemas agroflorestais. Tese (Doutorado em Solos e Nutrição de Plantas) - UFV.

Brasil. (1972). Levantamento exploratório-reconhecimento de solos do estado da Paraíba. https://library.wur.nl/isric/fulltext/isricu_i00003046_001.pdf

Bravo, K., Toselli, M., Baldi, E., Marcolini, G., Sorrenti, G, Quartieri, M., Marangoni, B. (2012) Effect of organic fertilization on carbon assimilation and partitioning in bearing nectarine trees. Scientia Horticultura, 137, 100-106 10.1016/j.scienta.2012.01.030

Brinkman, R. (1970). Ferrolysis, a hydromorphic, soil forming process. Geoderma, 3, 199-206 10.1016/0016-7061(70)90019-4

Bland, W., Rolls, D. (1998). Weathering: An introduction of the scientific principles. Routledge, 271. 10.4324/9781315824918

Carneiro, M. A. C., Souza, E. D. De, Reis, E. F. Dos, Pereira, H. S., Azevedo, W. R. de. (2009). Atributos físicos, químicos e biológicos de solo de cerrado sob diferentes sistemas de uso e manejo. Revista Brasileira de Ciência do Solo, 33, 147-157 10.1590/S0100-06832009000100016

Clemente, R., Walker, D. J., Pardo, T., Martínez-Fernández, D., Bernal, M. P. (2012). The use of a halophytic plant species and organic amendments for the remediation of a trace elements-contaminated soil under semi-arid conditions. Journal of Hazardous Materials, 223-224, 63-71 10.1016/j.jhazmat.2012.04.048

EMBRAPA - Empresa Brasileira de Pesquisa Agropecuária (2011). Manual de métodos de análise de solo. EMBRAPA Solos (2a ed.).

EMBRAPA - Empresa Brasileira de Pesquisa Agropecuária. (2013). Sistema Brasileiro de Classificação de Solos. EMBRAPA Solos, (3a ed.).

Farias, D. R., Oliveira, F. H. T., Santos, D., Arruda, J. A., Hoffman, R. B., Novais, R. F. (2009). Fósforo em solos representativos do estado da Paraíba: Isotermas de adsorção e medidas do fator capacidade de fósforo. Revista Brasileira de Ciência do Solo, 33, 623-632 10.1590/S0100-06832009000300015

Ferreira, M. M. (2010). Caracterização física do solo. In: Van Lier, Q. J. (Ed.). Física do Solo, Sociedade Brasileira de Ciência do Solo, 1-27.

Guareschi, R. F., Pereira, M. G., Perin, A. (2013). Frações da matéria orgânica em áreas de Latossolo sob diferentes sistemas de manejo no Cerrado do estado de Goiás. Semina: Ciências Agrárias, 34, 2615-2628 10.5433/1679-0359.2013v34n6p2615

Jakelaitis, A., Silva, A. A., Santos, J. B., Vivian, R. (2008). Qualidade da camada superficial de solo sob mata, pastagens e áreas cultivadas. Pesquisa Agropecuária Tropical, 38, 118-127 10.5216/pat.v38i2.4171

Jiménez Becker, S., Ebrahimzadeh, A., Plaza Herrada, B. M., Lao, M. T. (2010). Characterization of compost based on crop residues: changes in some chemical and physical properties of the soil after applying the compost as organic amendment. Communications in Soil Science and Plant Analysis, 41, 696-708 $10.1080 / 00103620903563931$ 
Kämpf, N., Curi, N. (2012). Formação e evolução do solo (Pedogênese). In: Ker, J. C., Curi, N., Schaefer, C. E. G. R., Vidal-Torrado, P. Pedologia: Fundamentos. Viçosa: Sociedade Brasileira de Ciência do Solo, 207-302.

Kiehl, E. J. (1979). Manual de edafologia: relações solo-planta. Ceres.

Lima Neto, J. A., Ribeiro, M. R., Corrêa, M. M., Souza Júnior, V. S., Lima, J. F. W. F., Ferreira, R. F. A. L. (2009). Caracterização e gênese do caráter coeso em Latossolos Amarelos e Argissolos dos Tabuleiros Costeiros do Estado de Alagoas. Revista Brasileira de Ciência do Solo, 33, 1001-1011 10.1590/S010006832009000400024

Madureira Filho, J. B., Atencio, D., McReath, I. (2000). Minerais e Rochas: constituintes da Terra sólida. In: Teixeira, W., Fairchild, T. R., Toledo, M. C. M. D., Taioli, F. Decifrando a terra. (2a ed.). Editora Nacional, 27-42.

Malavolta, E. (2006). Manual de nutrição mineral de plantas. Agronômica Ceres.

Mota, J. C. A., Assis Júnior, R. N. De, Amaro Filho, J., Libardi, P. L. (2008). Algumas propriedades físicas e hídricas de três solos na Chapada do Apodi, RN, cultivados com melão. Revista Brasileira de Ciência do Solo, 32, 49-58 10.1590/S0100-06832008000100006

Portugal, A. F., Costa, O. D. V., Costa, L. M. (2010). Propriedades físicas e químicas do solo em áreas com sistemas produtivos e mata na Região da Zona da Mata Mineira. Revista Brasileira de Ciência do Solo, 34, 575-585 10.1590/S0100-06832010000200032

Rezende, J. O. (2000). Solos coesos dos Tabuleiros Costeiros: Limitações agrícolas e manejo. Salvador: SEAGRI, (Série Estudos Agrícolas, 1).

Ribeiro, M. R., Oliveira, L. B., Araújo Filho, J. C. (2012). Caracterização morfológica do solo. In: Ker, J. C., Schaefer, C. E. G. R., Vidal-Torrado, P. (Eds.). Pedologia: fundamentos. Viçosa: Sociedade Brasileira de Ciência do Solo, 47-80.

Salton, J. C., Hernani, L. C. (1998). In: Salton, J. C., Hernani, L. C., Fonte, C. Z. Sistema de plantio direto. Brasília: Embrapa Agropecuária Oeste, 21-35.

Santos, A. C., Vilela, L. C., Barreto, P. M., Castro, J. G. D., Silva, J. E. C. (2010) Alterações de atributos químicos pela calagem e gessagem superficial com o tempo de incubação. Revista Caatinga, 23, 77-83 https://periodicos.ufersa.edu.br/index.php/caatinga/article/view/1327/pdf

Santos, H.G., Jacomine, P.K.T., Anjos, L.H.C., Oliveira, V.A., Lumbreras, J.F., Coelho, M. R., Almeida, J.A., Araujo Filho, J.C., Oliveira, J. B. e Cunha, T.J.F. Sistema Brasileiro de Classificação de Solos (5a ed.).Embrapa, 2018. 356 p. il. color.

Santos, J. C. B., Júnior Souza, V. S., Corrêa, M. M., Ribeiro, M, R., Almeida, M. C., Borges, L. E. P. (2012). Caracterização de neossolos regolíticos da região semiárida do estado de Pernambuco. Revista Brasileira de Ciência do Solo, 36, 683-695 10.1590/S0100-06832012000300001

Santos, R. D., Lemos, R. C., Santos, H. G., Ker, J. C., Anjos, L. H. C., Shimizu, S. H. (2013). Manual de descrição e coleta de solos no campo. Sociedade Brasileira de Ciência do Solo, 6. ed.

Shaffrath, V. R., Tormena, C. A., Fidalski, J., Gonçalves, A. C. A. (2008). Variabilidade e correlação especial de propriedades físicas de solo sob plantio direto e preparo convencional. Revista Brasileira de Ciência do Solo, 32, 1369-1377 10.1590/S0100-06832008000400001

Wastowski, A. D., Rosa, G. M., Cherubin, M. R., Rigon, J. P. G. (2010). Caracterização dos níveis de elementos químicos em solo submetido a diferentes sistemas de uso e manejo utilizando espectrometria de fluorescência de raios-X por energia dispersiva (EDXRF). Química Nova, 33, 1449-1452 10.1590/S010040422010000700005

Yamada, Y., Abdalla, S. R. S. (2004). A importância do potássio na produtividade e qualidade das colheitas e na sanidade das culturas é debatida em simpósio. http://www.ipni.net/publication/ia brasil.nsf/0/B7CE80534D402EB183257AA2005A1C52/\$FILE/Jornal107.pdf

Yao, R., Yang, J., Gao, P., Zhang, J., Jin, W. (2013). Determining minimum data set for soil quality assessment of typical salt-affected Farmland in the coastal reclamation area. Soil and Tillage Research, 128, 137-148 10.1016/j.still.2012.11.007 